\title{
BMJ Open Effect of multimorbidity patterns on the decline in health-related quality of life: a nationwide prospective cohort study in Japan
}

\author{
Takuya Aoki (D) ,,2 Shunichi Fukuhara, ${ }^{2,3,4}$ Yasuki Fujinuma, ${ }^{5}$ \\ Yosuke Yamamoto (iD) ${ }^{6,7}$
}

To cite: Aoki T, Fukuhara S, Fujinuma Y, et al. Effect of multimorbidity patterns on the decline in healthrelated quality of life: a nationwide prospective cohort study in Japan. BMJ Open 2021;11:e047812. doi:10.1136/ bmjopen-2020-047812

- Prepublication history and additional supplemental materials for this paper are available online. To view these files, please visit the journal online (http://dx.doi.org/10. 1136/bmjopen-2020-047812).

Received 10 December 2020 Accepted 28 May 2021

D Check for updates

(c) Author(s) (or their employer(s)) 2021. Re-use permitted under CC BY-NC. No commercial re-use. See rights and permissions. Published by BMJ.

For numbered affiliations see end of article.

\section{Correspondence to}

Professor Yosuke Yamamoto; yamamoto.yosuke.5n@kyoto-u. ac.jp

\section{ABSTRACT}

Objectives Longitudinal studies, which consider multimorbidity patterns, are useful for better clarifying the effect of multimorbidity on health-related quality of life (HRQoL) and for identifying the target population with poorer clinical outcomes among patients with multimorbidity. This study aimed to examine the effects of different multimorbidity patterns on the decline in HRQoL. Design Nationwide prospective cohort study.

Setting Japanese adult residents.

Participants Residents aged $\geq 50$ years selected by the quota sampling method.

Primary outcome measure Clinically relevant decline in $\mathrm{HRQOL}$ was defined as a $0.50 \mathrm{SD}$ (5-point) decrease in the 36-Item Short Form Health Survey (SF-36) component summary scores for 1 year.

Results In total, 1211 participants completed the followup survey. Among the multimorbidity patterns identified using confirmatory factor analysis, multivariable logistic regression analyses revealed that high cardiovascular/ renal/metabolic and malignant/digestive/urologic pattern scores were significantly associated with the clinically relevant decline in SF-36 physical component summary score (adjusted OR (aOR) $=1.25,95 \% \mathrm{Cl}: 1.08$ to 1.44 and $\mathrm{aOR}=1.28,95 \% \mathrm{Cl}: 1.04$ to 1.58 , respectively). High cardiovascular/renal/metabolic pattern score was also significantly associated with the clinically relevant decline in SF-36 role/social component summary score (aOR=1.23, 95\% Cl: 1.06 to 1.42 ).

Conclusions Our study revealed that multimorbidity patterns have different effects on the clinically relevant decline in $H R Q O L$ for 1 year. These findings can be useful in identifying populations at high risk and with poor clinical outcomes among patients with chronic diseases and multimorbidity for efficient resource allocation.

\section{INTRODUCTION}

Multimorbidity, usually defined as the coexistence of two or more chronic health conditions in an individual, is becoming an important issue across various healthcare settings including primary care, and its occurrence increases with age. ${ }^{1}$ The prevalence of multimorbidity is reported as $29.9 \%$ in adults,
Strengths and limitations of this study

- This is the first longitudinal study examining the effects of different multimorbidity patterns on healthrelated quality of life (HRQOL) decline.

- Multimorbidity patterns in the Japanese general population were generated using a confirmatory statistical approach, not an exploratory approach.

- Self-reported data on chronic health conditions may have introduced misclassification bias and selection bias.

- The effects of multimorbidity patterns on HRQoL decline over a long period, which are still unknown.

with the proportion increasing to $62.8 \%$ in those aged 65 years or older in the Japanese population. $^{2}$ It has become common for people to have multiple coexisting chronic health conditions. Therefore, identification of the target population with the presence of risk factors for poorer clinical outcomes is recommended in the guidelines on the assessment and management of patients with multimorbidity. ${ }^{3}$

A major challenge in risk assessments among patients with multimorbidity is identifying and classifying multimorbidity subtypes. ${ }^{4}$ Multimorbidity patterns, which are non-random cluster patterns of individual chronic health conditions, have currently gained increasing attention for a better understanding of the complex nature of the multimorbidity. In fact, there is a growing body of evidence showing that multimorbidity patterns have different relationships with clinical outcomes such as functional ability, depressive symptoms, polypharmacy and healthcare utilisation. ${ }^{25-7}$

Health-related quality of life (HRQoL) is one of the most important outcomes in patients with multimorbidity. ${ }^{8}$ A previous meta-analysis reported that HRQoL decreases 
with an increasing number of chronic health conditions ${ }^{9}$; however, only few longitudinal studies exist in the literature. Moreover, the findings of the associations between multimorbidity patterns and HRQoL are limited to few cross-sectional studies ${ }^{10-13}$ and longitudinal studies have never previously been conducted. Therefore, the relationship between the complex nature of multimorbidity and HRQoL remains unclear. Longitudinal studies, which consider multimorbidity patterns, can be useful for better clarifying the effect of multimorbidity on HRQoL and identifying the target population with poorer clinical outcomes among patients with multimorbidity. Nonrandom cluster patterns of health conditions may have a synergistic effect on HRQoL, and specific patterns may be related to the decline in HRQoL.

In the present study, we aimed to determine the effects of different multimorbidity patterns on the clinically relevant decline in HRQoL.

\section{METHODS}

\section{Design, setting and participants}

We conducted a nationwide prospective cohort study in Japan between December 2016 and December 2017. The quota sampling method was used to select possible participants, aged 16-84 years, from a resident panel administered by the Nippon Research Center. ${ }^{14}$ This large panel is composed of approximately 300000 residents who were selected by two-stage sampling method and responded to be willing to participate in surveys from the Nippon Research Center. In this study, quotas were set with regard to age, sex and residential area to make our sample representative of the demographic distribution across Japan, as shown in the most recent census data (online supplemental file 1). Data collection was either web based for participants aged $\leq 69$ years or mail based for those aged $\geq 70$ years. In total, 3307 participants completed baseline assessments using a self-administered questionnaire (mail survey response rate: $54.6 \%$ ). The questionnaire measured the presence of chronic health conditions, socioeconomic status and HRQoL. Among those who completed the baseline survey, participants aged $\geq 50$ years were eligible for this study. A follow-up questionnaire was distributed 12 months after the completion of the baseline survey to assess the change in HRQoL.

\section{Measures}

\section{Chronic health conditions}

In total, 15 chronic health conditions were assessed to identify multimorbidity patterns in this study. The following common chronic health conditions in the primary care setting were included in the analyses: hypertension, diabetes, dyslipidaemia, stroke, cardiac diseases (eg, coronary heart disease, heart failure and arrhythmia), respiratory diseases (eg, asthma, chronic obstructive pulmonary disease), digestive diseases (eg, gastro-oesophageal reflux disease, cirrhosis), kidney diseases (eg, chronic kidney disease), urologic diseases (eg, prostatic hypertrophy, overactive bladder), arthritis or rheumatism (eg, osteoarthritis, rheumatoid arthritis), lumbar diseases (eg, lumbar spinal stenosis, osteoporosis), neurological diseases (eg, epilepsy, dementia), mental disorders (eg, depression), malignancy and skin diseases (eg, atopic dermatitis). In a structured questionnaire, participants were asked if a doctor/nurse/paramedic had ever told them that they have each chronic health condition.

\section{HRQoL}

We used the Medical Outcomes Study 36-Item Short Form Health Survey (SF-36), which is one of the most widely used HRQoL measures worldwide. ${ }^{15} 16$ The SF-36 enables quantitative evaluation of subjective health status and its effect on daily physical, psychological and social functions. The SF-36 was translated into Japanese, ${ }^{17}$ and the Japanese version was validated for use in the general population. $^{18}$

The Japanese SF-36 was used to derive the physical component summary (PCS), mental component summary (MCS) and role/social component summary (RCS) scores as follows: after the eight subscale scores (physical functioning, role physical, role emotional, vitality, bodily pain, general health, social functioning and mental health) were calculated, a z-score was determined for each by subtracting the scale mean of a sample of the Japanese general population from an individual's scale score and then dividing by the SD from the Japanese general population. The products of the z-scores and factor scoring coefficients for the PCS were then summed together, and similar calculations were performed for the MCS and RCS. Each resulting sum was multiplied by 10 and added to 50 to calculate the component summary scores $\left(0-100\right.$ scale).$^{19}$

The primary outcome in this study was a clinically relevant decline in HRQoL for 1 year. The most widely used cut-offs for a minimally important change in HRQoL measures were very close to $0.50 \mathrm{SD} .{ }^{20}$ Therefore, in this study, we defined the clinically relevant decline in HRQoL as a $0.50 \mathrm{SD}$ (5-point) decrease in the SF-36 component summary scores.

\section{Covariates}

We included five covariates, namely, age, sex, years of education, household income and marital status, in the multiple regression analyses to adjust for potential confounders. All covariates were evaluated at the baseline survey using the self-administered questionnaire. Age was measured in years and categorised (50-59, 60-69, 70-79 and $\geqq 80$ years) for the analyses. All other covariates were measured as categorical variables.

\section{Statistical analysis}

We applied a two-step procedure to determine the associations between multimorbidity patterns and the clinically relevant decline in HRQoL. 


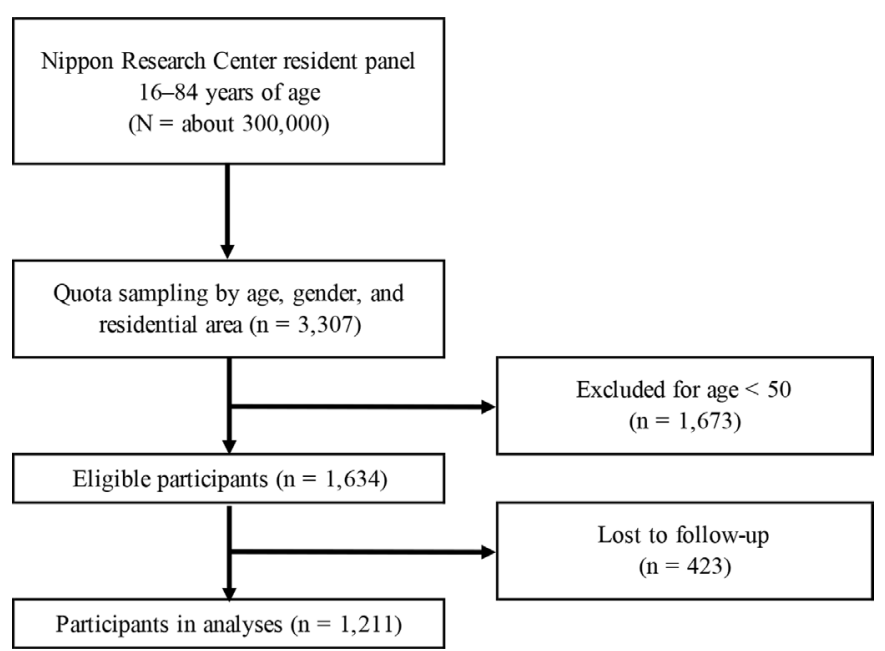

Figure 1 Participant flow chart.

Identification of multimorbidity patterns

In this study, we selected factor analysis as a statistical approach to identify multimorbidity patterns based on the theoretical rationale that multimorbidity pattern is conceptualised as a continuum of underlying pathological processes that manifest in a specific pattern of diseases for each person. ${ }^{4}$

In a previous study conducted in a Japanese population, five multimorbidity patterns were identified using exploratory factor analysis: cardiovascular/renal/metabolic, neuro/psychiatric, skeletal/articular/digestive, respiratory/dermal and malignant/digestive/urologic patterns. ${ }^{2}$ Exploratory factor analysis is one of the statistical approaches that is used to identify non-random cluster patterns in individual health conditions and categorise them into groups of multimorbid conditions; furthermore, the consistency in results across exploratory and confirmatory approaches support the robustness of identified multimorbidity patterns. Thus, we conducted a confirmatory factor analysis using the baseline morbidity data to further examine the multimorbidity patterns identified using exploratory factor analysis in the previous study in Japan. In the confirmatory factor analysis, we used the diagonally weighted least squares method because chronic health conditions were coded as dichotomous variables. Model fitness was assessed using the Comparative Fit Index (CFI), Tucker-Lewis Index (TLI), root mean square error of approximation (RMSEA) and standardised root mean square residual (SRMR). For CFI and TLI, a value of $>0.95$ indicates excellent goodness of fit. Previous studies have suggested that models with RMSEA $<0.07$ and SRMR $<0.08$ indicate a good fit. ${ }^{21}{ }^{22}$ For each participant, a multimorbidity pattern score was calculated for every identified pattern. These scores corresponded to the count of all health conditions in one pattern.

Associations of multimorbidity patterns with HRQoL

In the primary analysis of participants who completed the follow-up survey, multivariable logistic regression analyses were conducted to determine the effects of multimorbidity pattern scores on the clinically relevant decline in SF-36 component scores. In the secondary analysis of participants who completed the baseline survey, multivariable linear regression analyses were conducted to investigate the associations between multimorbidity pattern scores and baseline SF-36 component scores.

The following possible confounding variables measured at the baseline survey were included as covariates in the multivariable analyses: age, sex, years of education, household income and marital status. Each of the multimorbidity pattern scores was included individually in the model to avoid multicollinearity. Multicollinearity was avoided by ensuring variance inflation factor was less than 4 throughout the multivariable analyses. We performed 15 statistical tests in each primary and secondary analysis. For each analysis, statistical significance was set at $\mathrm{p}<0.05$ without adjustment for multiple comparisons. ${ }^{23}$ Missing data for independent variables were adjusted by applying multiple imputation, with 20 imputations, using a fully conditional specification. Statistical analyses were conducted using R, V.3.6.3 (R Foundation for Statistical Computing, Vienna, Austria; www.R-project.org).

\section{Patient and public involvement}

No patient involved.

\section{RESULTS}

Among the 1634 eligible participants aged $\geq 50$ years, 1211 (74.1\%) participants completed the follow-up survey (figure 1). Table 1 shows the baseline characteristics of the study population. There were no differences in sex, education level, annual household income, marital status, number of chronic health conditions, SF-36 PCS score and SF-36 RCS score between the 423 subjects who were lost to follow-up and those who had completed the follow-up. However, we noted a trend suggesting that subjects who were lost to follow-up were younger and had lower SF-36 MCS scores than those who completed the follow-up. Among the participants who completed the follow-up, the clinically relevant decline in HRQOL, which is defined as a 5-point decrease in the SF-36 component scores, was observed in 336 (27.7\%) participants with PCS, 231 (19.1\%) participants with MCS and 310 (25.6\%) participants with RCS.

Online supplemental file 2 shows the baseline frequencies of chronic health conditions. Hypertension was the most common diagnosis, followed by lumbar diseases and dyslipidaemia. There were no differences in the prevalence of chronic health conditions between subjects who responded to the baseline survey and those who had completed the follow-up.

Figure 2 shows the path diagrams of the confirmatory factor analysis to examine the multimorbidity patterns that were identified using an exploratory factor analysis in a previous study in Japan. The five patterns were cardiovascular/renal/metabolic, neuro/psychiatric, skeletal/ articular/digestive, respiratory/dermal and malignant/ 
Table 1 Participants' characteristics at baseline

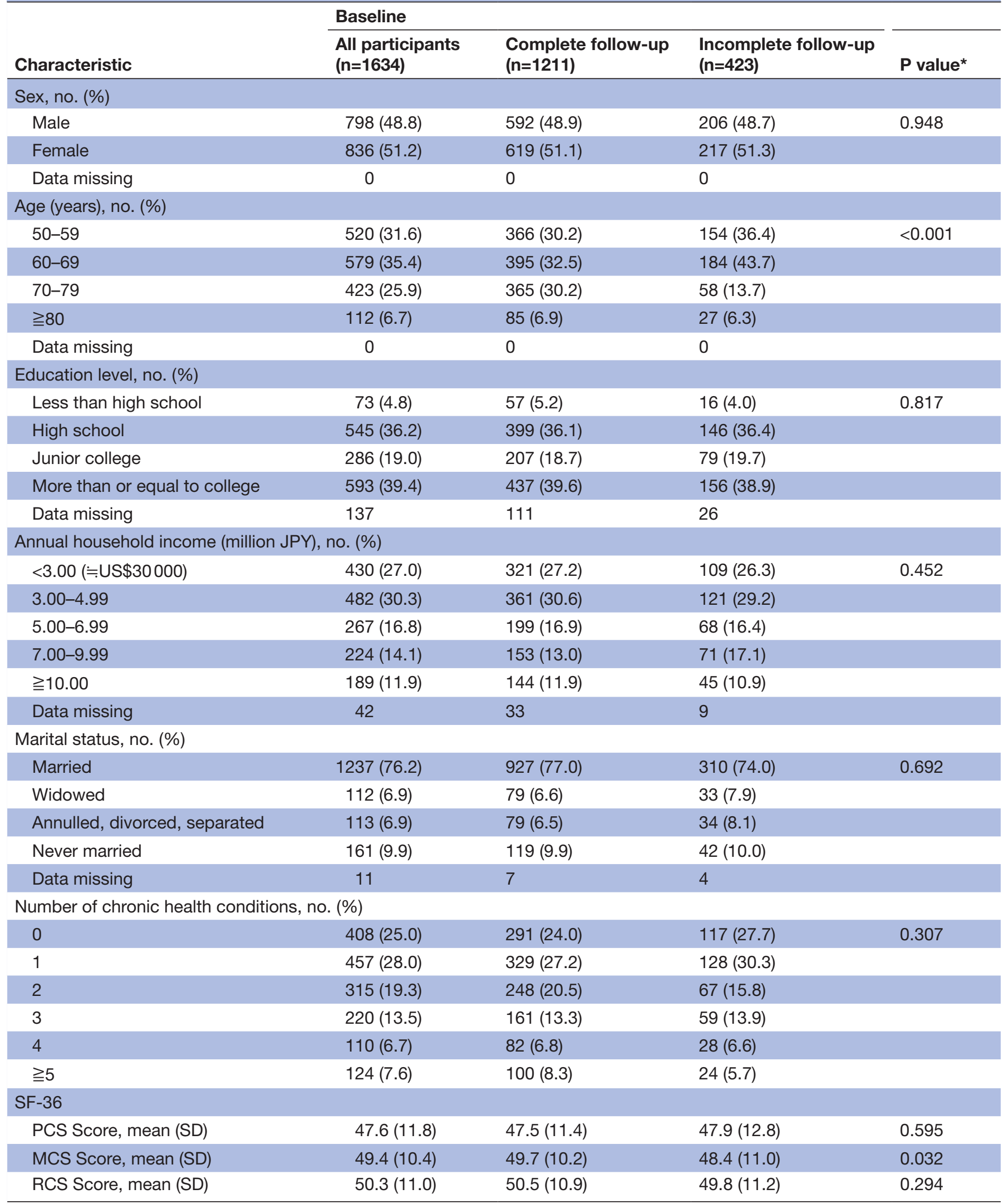

${ }^{*} \mathrm{P}$ value by t-test for continuous data and $\chi^{2}$ test for categorical data.

MCS, mental component summary; PCS, physical component summary; RCS, role/social component summary; SF-36, 36-Item Short Form Health Survey. 


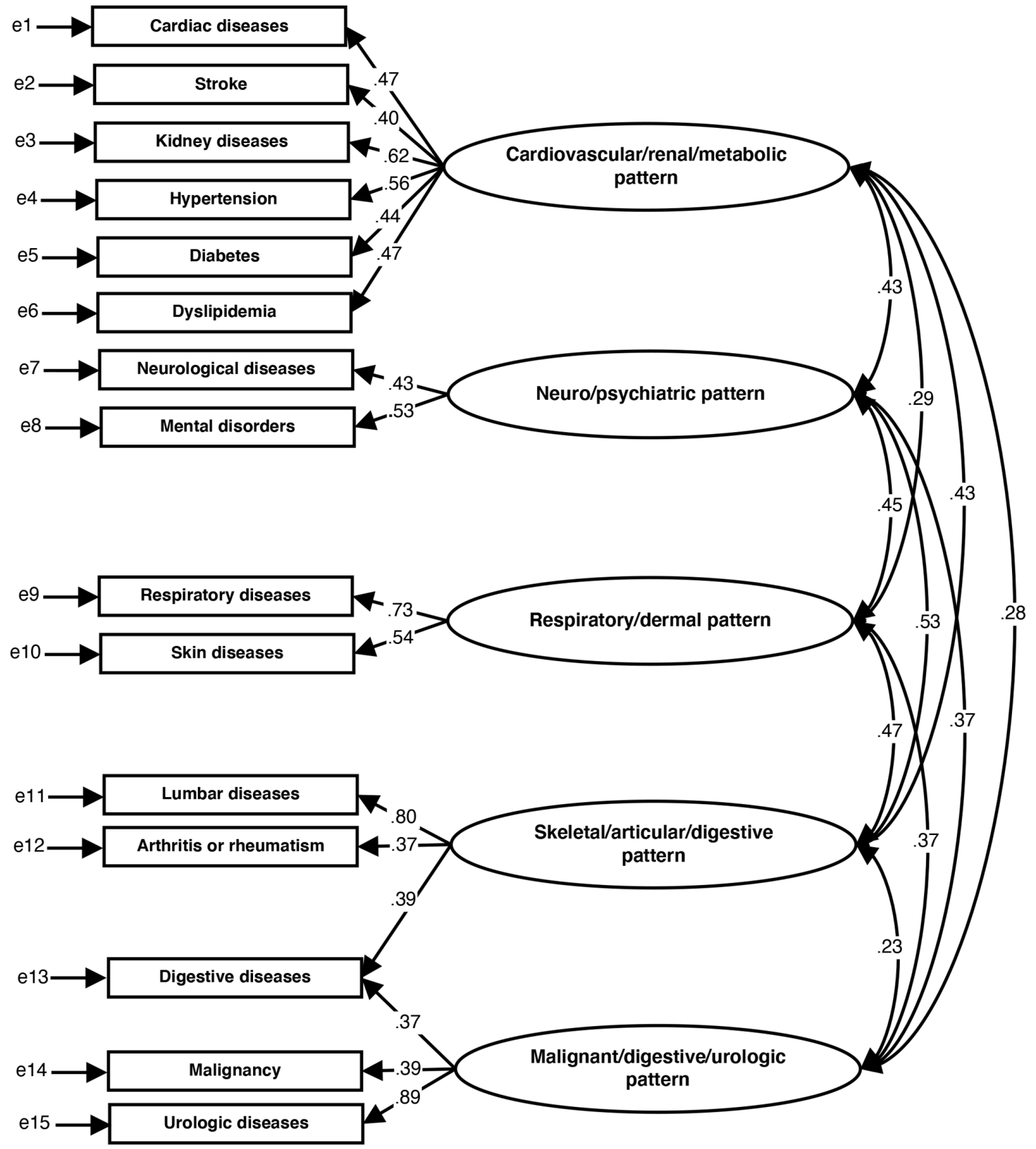

Figure 2 Multimorbidity patterns identified using confirmatory factor analysis. Squares are observed variables (conditions), ellipses are latent variables (patterns), values on the single-headed arrows are standardised factor loadings and values on the double-headed arrows are correlation coefficients.

digestive/urologic patterns. Factor loadings of each condition onto each pattern ranged from 0.37 to 0.89 . The correlation coefficients among patterns ranged from 0.23 to 0.53 . The conceptual model showed excellent goodness of fit, with CFI=0.980, TLI=0.973, RMSEA $<0.001$ and $\mathrm{SRMR}=0.066$.

Figure 3 shows the adjusted associations between multimorbidity pattern scores and the baseline SF-36 component summary scores. Skeletal/articular/digestive score had the strongest association with low PCS score (adjusted mean difference $=-4.38,95 \%$ CI: -5.14 to -3.62 ). Neuro/ psychiatric score had the strongest association with low MCS and RCS scores (adjusted mean difference $=-6.55$,
95\% CI: -8.61 to -4.49 , adjusted mean difference $=-5.31$, 95\% CI: -7.57 to -3.05 , respectively).

Figure 4 shows the adjusted associations between multimorbidity pattern scores and clinically relevant declines in SF-36 component scores. Cardiovascular/ renal/metabolic and malignant/digestive/urologic scores were significantly associated with the clinically relevant decline in PCS (adjusted OR (aOR)=1.25, $95 \%$ CI: 1.08 to 1.44 and $\mathrm{aOR}=1.28,95 \% \mathrm{CI}: 1.04$ to 1.58 , respectively). Cardiovascular $/ \mathrm{renal} / \mathrm{metabolic}$ score was also significantly associated with the clinically relevant decline in RCS (aOR=1.23, 95\% CI: 1.06 to 1.42 ). The associations of each multimorbidity pattern score 


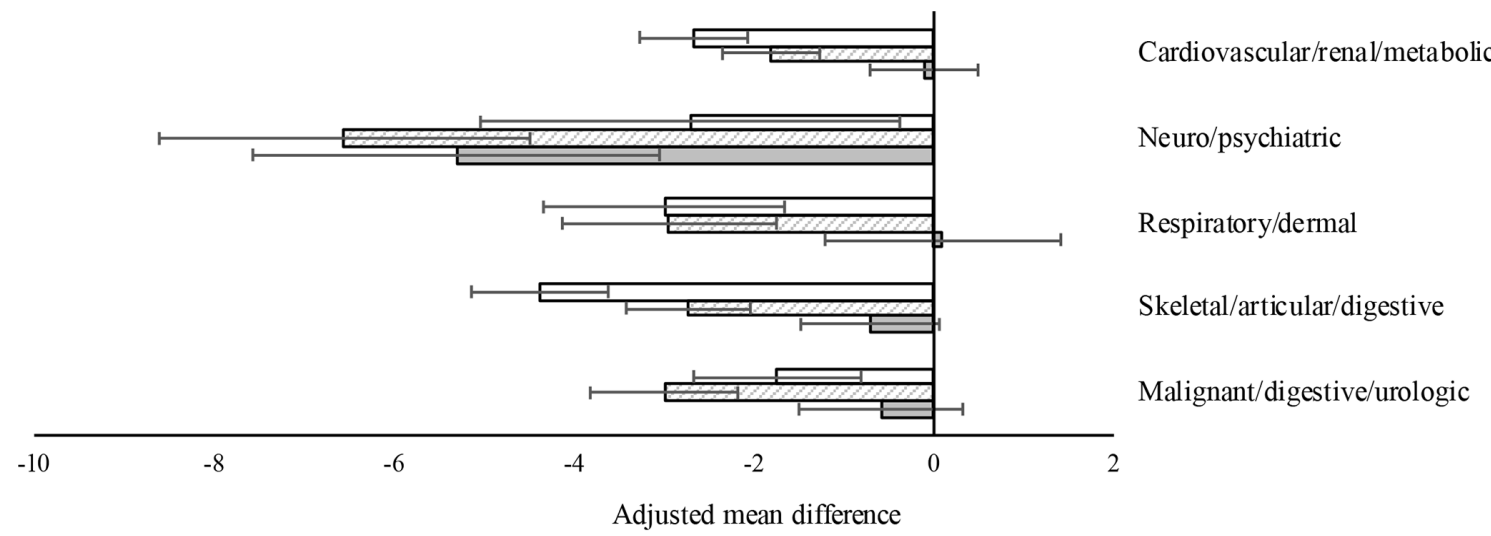

口PCS $\square$ MCS $\square$ RCS

Figure 3 Associations between multimorbidity pattern scores and baseline 36-Item Short Form Health Survey component summary scores. Multimorbidity pattern scores corresponded to the count of all health conditions in one pattern. Each pattern score was included individually in the model, adjusted for age, sex, years of education, household income and marital status. Error bars indicate 95\% Cls. MCS, mental component summary; PCS, physical component summary; RCS, role/social component summary.

with the clinically relevant decline in MCS were not significant.

\section{DISCUSSION}

Our nationwide prospective cohort study of the Japanese general population aged $\geq 50$ years revealed that multimorbidity patterns have different effects on the clinically relevant decline in SF-36 component scores for 1 year. After adjusting for covariates, high cardiovascular/renal/metabolic and malignant/digestive/ urologic pattern scores were found to be significantly associated with the clinically relevant decline in SF-36 PCS. High cardiovascular/renal/metabolic pattern score was also significantly associated with the clinically relevant decline in SF-36 RCS.

Among identified multimorbidity patterns in the present study, cardio/metabolic, psychiatric and musculoskeletal conditions were replicable multimorbidity patterns in previous systematic reviews. ${ }^{42}$

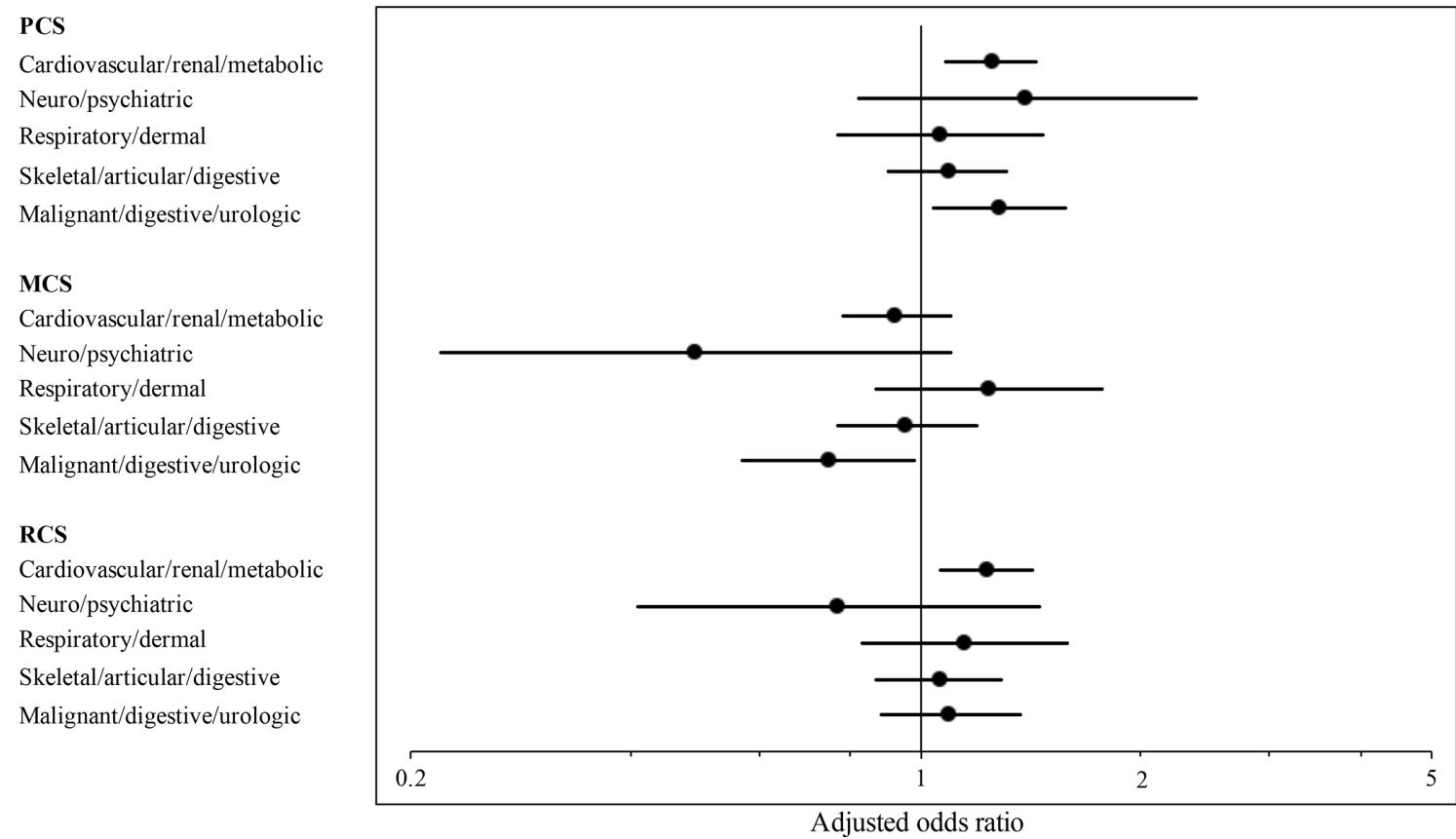

Figure 4 Effects of multimorbidity pattern scores on clinically relevant declines in 36-Item Short Form Health Survey component summary scores. Multimorbidity pattern scores corresponded to the count of all health conditions in one pattern. Each pattern score was included individually in the model, adjusted for age, sex, years of education, household income and marital status. Error bars indicate $95 \%$ Cls. MCS, mental component summary; PCS, physical component summary; RCS, role/ social component summary. 
We also identified the respiratory/dermal pattern, for example, a combination of asthma and atopic dermatitis is well established as the atopic march. ${ }^{25}$ In addition, the high prevalence of gastrointestinal (eg, colon) and urinary (eg, prostate) systems as primary sites in cancer survivors may have been a reason for the malignant/ digestive/urologic pattern. ${ }^{26}$

A few longitudinal studies have investigated the effect of multimorbidity on HRQoL. ${ }^{9}$ The current study examined the effect of multimorbidity patterns identified by a confirmatory statistical approach on the clinically relevant decline in HRQoL. The HRQoL decline over a relatively short period of 1 year, which indicates unstable health status, is an important clinical outcome in the management of chronic diseases and multimorbidity. Therefore, the findings of this study can be useful in identifying populations at high risk and with poor outcomes among patients with chronic diseases and multimorbidity for efficient resource allocation.

In the malignant pattern, progression or recurrence of the malignancy may affect the decline in physical HRQoL. In the cardiovascular/renal/metabolic pattern, there is a possibility that physical and social HRQoL may be deteriorated through increased treatment burden such as medication intake, drug management, self-monitoring, visits to the physician, laboratory tests, lifestyle changes and administrative tasks to access and coordinate care. ${ }^{27}$ Consistent with this hypothesis, in a previous study, we demonstrated that cardiovascular/renal/metabolic pattern had the strongest association with excessive polypharmacy, which can induce treatment burden. ${ }^{2}$ In addition, hospitalisation due to acute exacerbations of chronic heart failure and chronic kidney disease and the occurrence of new vascular events may reduce HRQoL in a short period of time. ${ }^{28} 29$

In our secondary analysis of the baseline SF-36 component scores, consistent with the findings of a previous study, the psychiatric pattern had the strongest association with poor mental HRQoL. ${ }^{10}$ This pattern also had the strongest association with poor social HRQoL, which has not been investigated before. However, the associations of psychiatric pattern with the clinically relevant decline in HRQoL were not significant. HRQoL in patients with neuro/psychiatric pattern might not decline significantly in the short term because they might have the opportunity to receive social support to increase resilience because of low mental and social HRQoL at baseline. ${ }^{30}$

To the best of our knowledge, this is the first longitudinal study examining the effects of different multimorbidity patterns on HRQoL decline. A key strength of our study is the use of data from a nationwide database, with a sample representative of the Japanese general population aged $\geq 50$ years, which allows for generalisation of its results to the wider population. In addition, multimorbidity patterns in the Japanese general population were generated using a confirmatory statistical approach, not an exploratory approach.

However, our study had several potential limitations. First, while the quota sampling method ensures that the sample is representative of the quota-defining characteristics, other characteristics might be disproportionately represented in the sample group. In addition, the web-based survey for participants aged $\leq 69$ years was completed only by those who were literate and who had access to the internet. Therefore, some selection bias may have affected our results. Second, we had a moderate follow-up rate of $74.1 \%$. We did not have complete outcome data on participants who had lost their ability to respond to the survey or who had died during the follow-up period. There was no significant difference in chronic health conditions, SF-36 PCS score and SF-36 RCS score between the participants who were lost to follow-up and those who completed it. However, middle-aged participants are often working, and participants with lower SF-36 MCS scores tend to have psychological symptoms, which might have been the reason why their response rate in the follow-up survey was lower. The participants who were lost to follow-up might have experienced a more frequent decline in HRQoL than those who completed the follow-up. Third, although self-reported data are commonly used to identify multimorbidity patterns in the general population, this method of assessment may have introduced misclassification bias and selection bias. Indeed, the sample of participants did not include patients with diseases such as advanced dementia. In addition, most health conditions represented affected organ systems and not specific diseases. Although assessing chronic health conditions affecting different organ systems was suggested to be useful in identifying patients with greater health needs in the previous study, ${ }^{31}$ this assessment might have influenced multimorbidity patterns identified in the present study. Fourth, the large number of combinations of multimorbidity patterns and SF-36 component scores raise the possibility of false-positive findings due to multiple testing. Finally, we investigated the decline in HRQoL over a short period of 1 year, and the effects of multimorbidity patterns on HRQoL decline over a long period are still unknown.

\section{CONCLUSION}

We found that multimorbidity patterns have different effects on the clinically relevant decline in HRQoL. Among the patterns, high cardiovascular/renal/metabolic and malignant/digestive/urologic pattern scores were significantly associated with a clinically relevant decline in physical HRQoL. High cardiovascular/renal/ metabolic pattern scores were significantly associated with a clinically relevant decline in social HRQoL. These findings are useful in identifying target populations at high risk and with poor clinical outcomes among patients with chronic diseases and multimorbidity.

\section{Author affiliations}

${ }^{1}$ Division of Clinical Epidemiology, Research Center for Medical Sciences, The Jikei University School of Medicine, Tokyo, Japan

${ }^{2}$ Section of Clinical Epidemiology, Department of Community Medicine, Graduate School of Medicine, Kyoto University, Kyoto, Japan

${ }^{3}$ Department of General Medicine, Shirakawa Satellite for Teaching And Research (STAR), Fukushima Medical University, Fukushima, Japan 
${ }^{4}$ Center for Innovative Research for Communities and Clinical Excellence (CIRC2LE), Fukushima Medical University, Fukushima, Japan

${ }^{5}$ Centre for Family Medicine Development, Japanese Health and Welfare Cooperative Federation, Tokyo, Japan

${ }^{6}$ Department of Healthcare Epidemiology, School of Public Health in the Graduate School of Medicine, Kyoto University, Kyoto, Japan

${ }^{7}$ Institute for Health Outcomes and Process Evaluation Research (iHope International), Kyoto, Japan

Contributors All authors contributed to the conception or design of the work, reviewed and edited the manuscript, contributed to the discussion of the data, performed critical review of the manuscript and gave the final approval of the manuscript before submission. TA performed the statistical analyses and drafted the manuscript. TA, YF and YY interpreted the analyses.

Funding The authors have not declared a specific grant for this research from any funding agency in the public, commercial or not-for-profit sectors.

Competing interests None declared.

Patient consent for publication Not required.

Ethical approval The Institutional Review Board of the Institute for Health Outcomes and Process Evaluation Research (iHope International) provided ethical approval for this study (approval number 201611) and the study was conducted in accordance with the Declaration of Helsinki.

Provenance and peer review Not commissioned; externally peer reviewed.

Data availability statement Due to the nature of this research, participants of this study did not agree for their data to be shared publicly, so supporting data are not available.

Supplemental material This content has been supplied by the author(s). It has not been vetted by BMJ Publishing Group Limited (BMJ) and may not have been peer-reviewed. Any opinions or recommendations discussed are solely those of the author(s) and are not endorsed by BMJ. BMJ disclaims all liability and responsibility arising from any reliance placed on the content. Where the content includes any translated material, BMJ does not warrant the accuracy and reliability of the translations (including but not limited to local regulations, clinical guidelines, terminology, drug names and drug dosages), and is not responsible for any error and/or omissions arising from translation and adaptation or otherwise.

Open access This is an open access article distributed in accordance with the Creative Commons Attribution Non Commercial (CC BY-NC 4.0) license, which permits others to distribute, remix, adapt, build upon this work non-commercially, and license their derivative works on different terms, provided the original work is properly cited, appropriate credit is given, any changes made indicated, and the use is non-commercial. See: http://creativecommons.org/licenses/by-nc/4.0/.

\section{ORCID iDs}

Takuya Aoki http://orcid.org/0000-0002-8232-2155

Yosuke Yamamoto http://orcid.org/0000-0003-1104-2612

\section{REFERENCES}

1 Wallace E, Salisbury C, Guthrie B, et al. Managing patients with multimorbidity in primary care. BMJ 2015;350:h176.

2 Aoki T, Yamamoto Y, Ikenoue T, et al. Multimorbidity patterns in relation to polypharmacy and dosage frequency: a nationwide, crosssectional study in a Japanese population. Sci Rep 2018;8:3806.

3 Muth C, Blom JW, Smith SM, et al. Evidence supporting the best clinical management of patients with multimorbidity and polypharmacy: a systematic guideline review and expert consensus. J Intern Med 2019;285:272-88.

4 Busija L, Lim K, Szoeke C, et al. Do replicable profiles of multimorbidity exist? systematic review and synthesis. Eur J Epidemiol 2019;34:1025-53.

5 Jackson CA, Jones $M$, Tooth L, et al. Multimorbidity patterns are differentially associated with functional ability and decline in a longitudinal cohort of older women. Age Ageing 2015;44:810-6.
6 Aoki T, Yamamoto Y, Shimizu S, et al. Physical multimorbidity patterns and depressive symptoms: a nationwide cross-sectional study in Japan. Fam Med Community Health 2020;8:e000234.

7 Gimeno-Miguel A, Gracia Gutiérrez A, Poblador-Plou B, et al. Multimorbidity patterns in patients with heart failure: an observational Spanish study based on electronic health records. BMJ Open 2019;9:e033174.

8 Smith SM, Wallace E, Salisbury C, et al. A core outcome set for multimorbidity research (COSmm). Ann Fam Med 2018;16:132-8.

9 Makovski TT, Schmitz S, Zeegers MP, et al. Multimorbidity and quality of life: systematic literature review and meta-analysis. Ageing Res Rev 2019;53:100903.

10 Walker V, Perret-Guillaume C, Kesse-Guyot E, et al. Effect of multimorbidity on health-related quality of life in adults aged 55 years or older: results from the SU.VI.MAX 2 cohort. PLoS One 2016;11:e0169282.

11 She R, Yan Z, Jiang H, et al. Multimorbidity and health-related quality of life in old age: role of functional dependence and depressive symptoms. J Am Med Dir Assoc 2019;20:1143-9.

12 Larsen FB, Pedersen $\mathrm{MH}$, Friis $\mathrm{K}$, et al. A latent class analysis of multimorbidity and the relationship to socio-demographic factors and health-related quality of life. A national population-based study of 162,283 Danish adults. PLoS One 2017;12:e0169426.

13 Zheng DD, McCollister KE, Christ SL, et al. Chronic condition patterns in the US population and their association with health related quality of life. Prev Med 2020;136:106102.

14 Nippon Research Center. Nippon research center. Available: https:// www.nrc.co.jp/english/index.html [Accessed 16 Jul 2020].

15 Stewart AL, Hays RD, Ware JE. The mos short-form general health survey. reliability and validity in a patient population. Med Care 1988;26:724-36.

16 Tarlov AR, Ware JE, Greenfield S, et al. The medical outcomes study. An application of methods for monitoring the results of medical care. JAMA 1989;262:925-34.

17 Fukuhara S, Bito S, Green J, et al. Translation, adaptation, and validation of the SF-36 health survey for use in Japan. J Clin Epidemiol 1998;51:1037-44.

18 Fukuhara S, Ware JE, Kosinski M, et al. Psychometric and clinical tests of validity of the Japanese SF-36 health survey. J Clin Epidemiol 1998;51:1045-53.

19 Ware JE, Kosinski M, Dewey JE. How to score version 2 of the SF-36 health survey. Boston: QualityMetric, 2000.

20 Norman GR, Sloan JA, Wyrwich KW. Interpretation of changes in health-related quality of life. Med Care 2003;41:582-92.

$21 \mathrm{Hu}$ Li-tze, Bentler PM. Cutoff criteria for fit indexes in covariance structure analysis: conventional criteria versus new alternatives. Struct Equ Modeling 1999;6:1-55.

22 Steiger $\mathrm{JH}$. Understanding the limitations of global fit assessment in structural equation modeling. Pers Individ Dif 2007;42:893-8.

23 Rothman KJ. No adjustments are needed for multiple comparisons. Epidemiology 1990;1:43-6.

24 Prados-Torres A, Calderón-Larrañaga A, Hancco-Saavedra J, et al Multimorbidity patterns: a systematic review. J Clin Epidemiol 2014;67:254-66.

25 Spergel JM. From atopic dermatitis to asthma: the atopic March. Ann Allergy Asthma Immunol 2010;105:99-106.

26 Hewitt M, Rowland JH, Yancik R. Cancer survivors in the United States: age, health, and disability. J Gerontol A Biol Sci Med Sci 2003;58:M82-91.

27 Tran V-T, Harrington M, Montori VM, et al. Adaptation and validation of the treatment burden questionnaire (TBQ) in English using an Internet platform. BMC Med 2014;12:109.

28 Albuquerque de Almeida F, Al MJ, Koymans R, et al. Impact of hospitalisation on health-related quality of life in patients with chronic heart failure. Health Qual Life Outcomes 2020;18:262.

29 Nishikawa K, Takahashi K, Yamada R, et al. Influence of chronic kidney disease on hospitalization, chronic dialysis, and mortality in Japanese men: a longitudinal analysis. Clin Exp Nephrol 2017;21:316-23.

30 Ozbay F, Johnson DC, Dimoulas E, et al. Social support and resilience to stress: from neurobiology to clinical practice. Psychiatry 2007;4:35-40.

31 Harrison C, Britt H, Miller G, et al. Examining different measures of multimorbidity, using a large prospective cross-sectional study in Australian general practice. BMJ Open 2014;4:e004694. 